\title{
Comparative study of seven commercial yeast identification systems
}

\author{
P E Verweij, I M Breuker, A J M M Rijs, J F G M Meis
}

\begin{abstract}
Aims-To compare the performance of seven commercial yeast identification methods with that of a reference method, and to compare the costs of the commercial kits.

Methods-Clinical yeast isolates $(n=52)$, comprising 19 species, were identified using Vitek, Api ID 32C, Api 20C AUX, Yeast Star, Auxacolor, RapID Yeast Plus system, and Api Candida and compared with a reference method which employed conventional tests.

Results-The percentage of correctly identified isolates varied between $59.6 \%$ and $80.8 \%$. Overall, the highest performance was obtained with Api Candida (78.8\%) and Auxacolor (80.8\%). Among germ tube negative yeast isolates, Auxacolor and Api Candida both identified 93.1\% of isolates correctly. All systems failed to identify $C$ norvegensis, $C$ catenulata, C haemulonii, and $C d u b-$ liniensis. In comparison with Auxacolor, the Api Candida is less expensive and requires less bench time.

Conclusions-Auxacolor and Api Candida appeared to be the most useful systems for identification of germ tube negative yeast isolates in clinical microbiology laboratories, although one should be aware that several germ tube negative Candida species cannot be identified by these systems. (f Clin Pathol 1999;52:271-273)
\end{abstract}

Keywords: yeasts; commercial kits

Over the past decade the significance of infections by yeasts has increased, especially those caused by germ tube negative yeasts. ${ }^{1}$ Given the variable susceptibility of different species of yeasts to antifungal agents, the rapid and correct identification is of clinical importance. Furthermore, the identification of yeast isolates to species level makes it possible to study the epidemiology of colonisation and infection and the transmission of infections in hospitals. Conventional identification methods, however, are laborious and time consuming. Therefore, rapid commercially available identification methods have been developed which allow for identification of germ tube negative yeasts within 48 hours. In this study, we have evaluated the performance of seven commercial identification methods with clinical yeast isolates. The performance was determined by comparing the identification by each commercial kit with that by a reference method. The costs of the kits for routine identification were also compared.

\section{Methods}

ISOLATES

Fifty two clinical yeast and yeast-like fungal isolates were used in this study, including 19 species: Candida albicans ( $\mathrm{n}=7$ isolates), $C$ krusei (9), C glabrata (4), C guilliermondii (2), $C$ catenulata (2), $C$ haemulonii (1), $C$ inconspicua (2), $C$ kefyr (4), $C$ lusitaniae (2), $C$ magnoliae (1), $C$ dubliniensis (1), $C$ norvegensis (2), $C$ parapsilosis (3), C tropicalis (3), Cryptococcus neoformans (4), Geotrichum candidum (1), G capitatum (1), Rhodotorula rubra (1), and Saccharomyces cerevisiae (2). All isolates were cultured from patients admitted to the University Hospital Nijmegen. The selection of isolates was based on the distribution of genera and species recovered in routine clinical practice in our hospital rather than on the presence of the fungus in the database of the commercial kits. The isolates had been stored at $-70^{\circ} \mathrm{C}$ until use. After thawing, each isolate was subcultured onto Sabouraud chloramphenicol medium for control of purity of the isolates. One colony was subcultured on Sabouraud chloramphenicol agar plates and incubated at $30^{\circ} \mathrm{C}$.

\section{IDENTIFICATION SYSTEMS}

Isolates were identified by a reference method using conventional tests for assimilation of carbohydrates including D-galactose, D-xylose, maltose, cellobiose, lactose, raffinose, rhamnose, L-arabinitol, myo-inositol, 2-keto-Dgluconate, L-lactate, citrate, $\mathrm{L}$ and D-arabinose, D-gluconate, L-sorbose, sucrose, D-ribose, ribitol, xylitol, D-mannitol, nitrate, nitriet, ethylamine, and creatinine. Fermentation of glucose, resistance to cycloheximide, growth at $37^{\circ} \mathrm{C}$ and $42^{\circ} \mathrm{C}$, and macroscopic and microscopic morphology ${ }^{23}$ were also determined.

Seven commercial yeast identification kits were evaluated: Vitek (bioMérieux Vitek, Hazelwood, Missouri, USA), Api ID 32C (bioMérieux, Lyon, France), Api 20C AUX (bioMérieux, France), Yeast Star (CLARC Laboratories, Heerlen, The Netherlands), Auxacolor (Sanofi Diagnostics Pasteur, Marnes-La-Coquette, France), RapID Yeast Plus system (Innovative Diagnostic Systems, Norcross, Georgia, USA), and Api Candida (bioMérieux, France). Each system was used according to the manufacturer's instructions.

The Vitek card comprises of 30 wells which contain 26 biochemical broths and four negative control broths. The cards are incubated at $30^{\circ} \mathrm{C}$ 
Table 1 Performance of seven commercial identification systems with 52 clinical yeast isolates

\begin{tabular}{|c|c|c|c|c|c|c|}
\hline \multirow[b]{2}{*}{ Identification system } & \multicolumn{3}{|c|}{$\begin{array}{l}\text { All isolates }(n=52) \text { : } \\
\text { identification }(\%)\end{array}$} & \multicolumn{3}{|c|}{$\begin{array}{l}\text { Germ tube negative isolates }(n=29) \text { : } \\
\text { identification }(\%)\end{array}$} \\
\hline & Correct & Incorrect & Non & Correct & Incorrect & Non \\
\hline Vitek & 59.6 & 15.4 & 25.0 & 62.1 & 0 & 37.9 \\
\hline Api ID 32C & 63.5 & 34.6 & 1.9 & 72.4 & 27.6 & 0 \\
\hline Api 20C AUX & 59.6 & 36.5 & 3.9 & 62.1 & 37.9 & 0 \\
\hline Yeast Star & 59.6 & 40.4 & 0 & 79.3 & 20.7 & 0 \\
\hline Auxacolor & 80.8 & 7.7 & 11.5 & 93.1 & 0 & 6.9 \\
\hline RadID Yeast Plus & 76.9 & 11.5 & 11.5 & 86.2 & 3.5 & 10.3 \\
\hline Api Candida & 78.8 & 13.5 & 7.7 & 93.1 & 6.9 & 0 \\
\hline
\end{tabular}

for 24 to 48 hours. Colour changes are read spectrophotometrically and reported as a nine digit numerical code. The database includes 36 yeast species.

The ApiID 32C and 20C AUX contain 32 and 20 microtubes, respectively. The reactions are read visually after 24,48 , or 72 hours of incubation at $30^{\circ} \mathrm{C}$ by comparing the turbidity of the microtubes with that of controls. A numerical profile of 10 and seven digits, respectively, can be deduced. The databases of ApiID 32C includes 63 yeast species and that of Api 20C AUX, 43.

The Yeast Star system relies on the inhibitory effects of specific dyes on yeast growth. A panel of six dyes is placed on top of an inoculated solid growth medium and incubated for 24 to 48 hours at $37^{\circ} \mathrm{C}$. The results are recorded in the form of a six digit code. The database contains 16 yeast species.

The Auxacolor is an identification system based on carbohydrate utilisation, and growth is visualised by colour change of a $\mathrm{pH}$ indicator. The microtitre plate contains 16 wells and the plates are read after 24 to 72 hours of incubation at $30^{\circ} \mathrm{C}$. In addition, various other characteristics are used to complete the identification, such as the ability to grow at $37^{\circ} \mathrm{C}$, the formation of mycelium or arthrospores, and the presence of a capsule. The database contains 26 yeast species.

The RapID Yeast Plus system is based on utilisation of carbohydrate substrates, hydrolysis of fatty acids and urea, and enzymatic hydrolysis of glycoside and aryl-amide. A panel of 18 cavities is incubated for four hours at $30^{\circ} \mathrm{C}$ and read after adding specific reagents. A six digit code is derived from the results and compared to those of a database which includes 43 yeast species.

The Api Candida consists of 10 tubes containing dehydrated substrates and relies on sugar acidification or enzymatic reactions. The strips are read after 24 and 48 hours of incubation at $30^{\circ} \mathrm{C}$. A four digit numerical profile is obtained which is compared with those in a database with 26 yeast species.

ADDITIONAL TESTS

Additional tests are recommended in the case of low discrimination by all systems, and include morphological characteristics such as production of germ tubes, chlamydospores, colony morphology, presence of capsules, and ability to form mycelium, pseudomycelium, arthroconidia, or blastoconidia. For Api ID 32C, seven supplementary tests can be read.
The Auxacolor includes morphological characteristics in the numerical profile.

INTERPRETATION

A "correct identification" was defined as a numerical profile obtained with a system that was included in the manufacturer's database and in accordance with the identification by the reference method. Isolates that required additional tests to achieve a correct identification were also grouped in this category. Isolates that produced a numerical profile that was not listed in the manufacturer's database were classified as "non-identification." When the identification with the commercial system was not in accordance with that obtained by the reference method the result was classified as "incorrect identification."

STUDY DESIGN

The identification was performed in different institutes in The Netherlands and Belgium that used a commercial kit for routine identification, to ensure that the identification was performed by experienced personnel. The reference method, Auxacolor, and RapID Yeast Star Plus were performed at the University Hospital Nijmegen, Vitek at the University Hospital Rotterdam, Api ID 32 at Bronovo Hospital in The Hague, Api 20C AUX at Janssen Research Foundation in Beerse, Belgium, and Yeast Star at Catharina Hospital in Veldhoven, The Netherlands. The 52 yeast isolates were blinded and sent to each participating institute.

costs

The costs per test were calculated for each of the commercial kits and of additional tests if necessary. The mean bench time spent for identification was also calculated.

\section{Results}

The performance of each of the commercial identification systems is shown in table 1 . The performance was analysed for all yeasts and for the eight most frequently cultured germ tube negative yeasts at our hospital, which include C glabrata, C krusei, C kefyr, C lusitaniae, $C$ norvegensis, $C$ parapsilosis, $C$ tropicalis, and Saccharomyces cerevisiae (29 isolates). The proportion of correctly identified yeasts varied between $59.6 \%$ and $80.8 \%$. We had difficulties with turbidity reading with Api ID 32C and Api 20C AUX. In addition, colour change reading was sometimes difficult for RapID Yeast Plus. Additional tests were necessary with Vitek, Api 20C AUX, RapID Yeast Plus, and Api Candida. Furthermore, several germ tube negative yeasts which are regularly recovered from clinical specimens, such as $C$ norvegensis, were missing in the databases of Vitek, Yeast Star, and RapID Yeast Plus System. C norvegensis was among four yeast isolates that were not correctly identified by any of the systems. This was also the case for $C$ catenulata, $C$ haemulonii, and C maltosa. C haemulonii was not included in any of the manufacturers' databases and $C$ catenulata only in that of Api ID 32C. $C$ maltosa was misidentified by the reference 
Table 2 Comparison of costs and bench time required per test for seven commercial identification methods

\begin{tabular}{llll}
\hline Identification system & Cost/test $(£)$ & Bench time $(\mathrm{min})$ & Costidentification $\left(\mathcal{C}^{\prime}\right)$ \\
\hline Auxagram reference method & 1.92 & 18 & 4.74 \\
Vitek & 2.96 & 12 & 4.84 \\
Api ID 32C & 2.96 & 12 & 4.84 \\
Api 20C AUX & 2.87 & 12 & 4.76 \\
Yeast Star & 1.58 & 10 & 3.15 \\
Auxacolor & 4.44 & 7 & 5.54 \\
RapID Yeast Plus & 5.54 & 10 & 7.11 \\
Api Candida & 3.39 & 5 & 4.17 \\
\hline
\end{tabular}

method since additional tests showed that it was actually $C$ dubliniensis. None of the databases of the commercial identification systems included $C$ dubliniensis. The isolate was identified as $C$ albicans by Vitek and Api 20 AUX, as $C$ catenulata by Api ID 32C, and as Trichosporum cutaneum by Yeast Star. The Auxacolor, Api Candida, and RapID Yeast Plus system gave nonidentification.

Considerable variation of costs and bench time was observed for the identification systems (table 2).

\section{Discussion}

In most clinical microbiology laboratories commercial identification systems are used for identification of germ tube negative yeasts. The performance of commercial identification systems varied considerably. Overall, Auxacolor and Api Candida showed the highest percentage of correctly identified yeasts, including the germ tube negative yeasts. Auxacolor also showed the lowest percentage of incorrect identification. An advantage of the Auxacolor is that the isolates are not only identified by biochemical characteristics, but morphological appearance and ability to form mycelia are also taken into account, which reduces the need to perform additional tests. The system is, however, relatively expensive.

The identification rates of the kits evaluated in the present study were lower than those reported previously, which are greater than $80 \%{ }^{4-7}$ The identification rates, however, depend on the panel of yeasts which is used to evaluate the kit. The present study was designed to evaluate the performance of the kits in the setting of identification of isolates encountered routinely in a clinical microbiology laboratory. It is important to note that even kits that are considered reliable show lower identification rates in this setting, since yeast isolates are included which are not listed in the database of the kit. In the latter case a non-identification result is preferable to an incorrect identification.
$C$ dubliniensis has only recently been described as a cause of oropharyngeal candidosis in HIV positive patients. ${ }^{8} \mathrm{C}$ dubliniensis is difficult to distinguish from $C$ albicans since both species produce germ tubes and chlamydospores. In clinical microbiology laboratories yeast isolates that produce germ tubes and chlamydospores are considered to be $C$ albicans, and no additional tests are performed. However, both species can be differentiated if discriminative media, such as CHROMagar candida plates, are used for primary isolation. ${ }^{9}$ Tests such as abundant production of chlamydospores and the ability to grow at $45^{\circ} \mathrm{C}$ may also help to differentiate between the two species. ${ }^{10}$ The commercial identification systems presently available fail to identify this new species correctly. However, if a commercial system identifies a germ tube positive yeast as $C$ albicans, one should consider $C$ dubliniensis if the strain fails to utilise xylose or to elaborate $\beta$-glucosidase.

Independent of the system used, morphological examination of yeast isolates remains essential to obtain a correct identification; in addition, both Auxacolor and Api Candida are useful and rapid systems for the routine identification of germ tube negative yeasts.

We thank the consultant microbiologists Drs H Endtz, A Jansz, and A Horrevorts, and Prof F C Odds for identifying the yeast isolates.

1 Hazen KC. New and emerging yeast pathogens. Clin Microbiol Rev 1995;4:462-78.

2 Barnett JA, Payne RW, Yarrow D. Yeasts: characteristics and identification. Cambridge: Cambridge University Press, 1990.

3 Lodder J, Kreger-Van Rij NJW. The yeasts, a taxonomic study. Amsterdam: Elsevier, 1984.

4 Davey KG, Chant PM, Downer CS, et al. Evaluation of the Auxacolor system, a new method of clinical yeast identification. F Clin Pathol 1995;48:807-9.

5 Fricker-Hidalgo H, Vandapel O, Duchesne MA, et al. Comparison of the new API Candida system to the ID $32 \mathrm{C}$ system for identification of clinically important yeast species. $\mathcal{F}$ Clin Microbiol 1996;34:1846-8.

6 Buchaille L, Freydière, Guinet R, et al. Evaluation of six commercial systems for identification of medically important yeasts. Eur F Clin Microbiol Infect Dis 1998;17:479-88.

7 Ramani R, Gromadzki S, Pincus DH, et al. Efficacy of API20C and ID 32C systems for identification of common and rare clinical yeast isolates. F Clin Microbiol 1998;36: 3396-8.

8 Sullivan DJ, Westereng TJ, Hayes KA, et al. Candida dubliniensis sp. Nov.: phenotypic and molecular characteriHIV-infected individuals. Microbiology 1995;141:1507-21.

9 Schoofs A, Odds FC, Colebunders R, et al. Use of specialized isolation media for recognition and identification of Candida dubliniensis isolates from HIV infected patients. Eur f Clin Microbiol Infect Dis 1997;16:296-300.

10 Pinjon E, Sullivan D, Salkin I, et al. Simple, inexpensive, reliable method for differentiation of Candida dubliniensis from Candida albicans. F Clin Microbiol 1998;36:2093-5. 